\title{
Metabolic Bone Diseases of Captive Mammal, Reptile and Birds
}

\author{
Ranjan Kumar ${ }^{1 *}$, Tiwari RK, Rajeev Kumar Asthana, Pramod Kumar, Brajesh Shahi and Saha SK ${ }^{2}$ \\ ${ }^{1}$ Animal science,, Dr. Rajendra Prasad Central Agricultural University, India \\ ${ }^{2}$ Department of Animal Nutrition Division, India
}

*Corresponding author: Ranjan Kumar, Department of Agricultural Sciences, Bihar, India

Submission: 眥 February 24, 2018; Published: 眥 April 10, 2018

\section{Introduction}

Metabolic Bone Disease (MBD) is the collective name given to a number of symptoms and problems commonly seen in captive mammals, birds and reptiles. The captive housed indoor animals are more susceptible to MBD; because of they have little access to UV light. Other names for MBD include Fibrous Osteodystrophy and Secondary Nutritional Hyperparathyroidism. All vertebrates need certain nutritional building blocks to form their skeletal systems. When they do not get sufficient nutrition in the proper proportions to form healthy bone structure, the animal suffer from metabolic bone disease. Symptoms of metabolic bone disease vary between species and individual animals, but includes soft short jaws, sprawl (inability to lift the body off the ground), anorexia, lameness, swollen limbs, bone fractures, curvature of the spine, tremors, small or deformed carapaces and death. Metabolic bone disease is particularly devastating for growing animals since this is the time in their lives when they are most actively forming their skeletal structure. This disease is very common especially in captive mammal, reptile and birds due to nutritional and improper husbandry practices. Therefore an attempt to made through this mini-review paper to educate reptile and birds owners about proper husbandry particularly in dietary needs and care management, because these all nutritional disorders seen in captive animals can be preventable.

\section{Metabolic bone disease in captive mammals}

Captive primates housed indoors with little or no access to Ultraviolet (UV) light have historically been susceptible to metabolic bone disease. This was especially true of New World primates, who, unlike Old World primates, cannot effectively utilize the plant form of vitamin D (ergocalciferol or vitamin D2) [1]. Supplementing the diet of captive primates with vitamin $D_{3}$, the animal form of the vitamin, has largely proved effective at reducing the incidence of bone disease in primates. Cholecalciferol, commonly called vitamin $\mathrm{D}_{3}$ is a precursor to the steroid hormone calcitriol (1,25-dihydroxycholecalciferol, or 1,25- $(\mathrm{OH}) 2$-vitamin $\mathrm{D}_{3}$ ). In most vertebrates studied to date, cholecalciferol is produced in the skin by a two-stage process. First pro vitamin D (7-dehydrocholesterol) is converted to pre vitamin $\mathrm{D}$. This reaction requires irradiation of the skin by ultraviolet light in the wavelength range of $290-315 \mathrm{~nm}$, or UV-B (Holick et. al., 1981). Pre vitamin D is then converted to cholecalciferol (vitamin D) by a thermally dependent process. Vitamin D then enters the blood and binds to circulating proteins, primarily vitamin D-binding protein.

To form calcitriol, the vitamin D metabolite with the highest biological activity, cholecalciferol is first hydroxylated in the liver to form 25-(OH)-vitamin $\mathrm{D}_{3}$ or calcidiol, which is the main storage form of the hormone. Like vitamin D, calcidiol circulates in the blood primarily bound to vitamin D-binding protein. Hydroxylation of calcidiol in the kidney forms 1,25- $(\mathrm{OH}) 2$-vitamin $\mathrm{D}_{3}$. The hydroxylation of 25-(OH)-D to 1, $25(\mathrm{OH}) 2-\mathrm{D}$ is a tightly regulated physiological process. The known function of 1, 25-(OH)2-D is to regulate whole-body calcium metabolism. The hormone enhances intestinal absorption of both calcium and some phosphate, and acts on the kidneys to stimulate renal conservation of calcium and phosphate. It also has direct effects on bone; in concert with parathyroid hormone, calcitriol regulates the mobilization of calcium from bone. Adequate amounts of the hormone are necessary for normal growth and development of bone, and in maintaining mature bone tissue. Although there is little direct evidence that 1,25- $(\mathrm{OH}) 2-\mathrm{D}$ has biological functions in addition to whole-body calcium regulation, many factors suggest the possibility.

\section{Vitamin D and captive animals}

For most animals under natural conditions vitamin D is not a nutrient. It is an endogenously produced component of an endocrine-like hormonal system. However, many captive animals are kept under conditions in which they are exposed to little or no UV radiation. Thus they require an exogenous source of vitamin D.

\section{a. Primates at risk}

There are two categories of captive primates for which it appears to be difficult to maintain adequate vitamin D status by 
dietary means: mother-reared, nursing infants, and multiparous, callitrichids (specifically common marmosets).

\section{b. Nursing infants}

There are little information regarding level of vitamin D in milk. However, the concentrations of vitamin D in human milk (25 IU/L) [2] is too low to supply the suggested daily requirement for human infants (400 IU/day). Human infants fed solely on breast milk and without access to UV-B radiation would be at risk of developing rickets $[3,4]$. This appears to be true of non- human primates as well. Clinical rickets has been diagnosed in several colobus monkeys and a Francois langur (Morrisey et. al.), silvered leaf monkeys and sakis (R. Cambre pers. Comm.), and gorillas.

\section{c. Common marmosets}

Most New World monkeys (owl monkeys, genus Aotus, appear to be the only exception) differ from other primates in vitamin $\mathrm{D}$ metabolism. They cannot effectively utilize vitamin $D 2$ [1].

\section{d. Strategies for avoiding vitamin D deficiency}

The way of providing vitamin $\mathrm{D}$ through the diet for most animals has perpetuated the classification of this substance as a nutrient. For the many animals housed indoors at zoos and laboratory colonies, this classification has become the practical truth. However, we are beginning to appreciate that there may be significant variation among species in the efficiency with which dietary vitamin D can meet requirements. With the increase in large, indoor, multispecies exhibits in zoos this issue becomes increasingly germane. Levels of cholecalciferol necessary for meeting the requirements of callitrichids present the danger of toxicity for other animals in the same exhibit [5]. Nursing infants that are not yet eating other foods may not benefit from maternal dietary supplementation. Thus, there are many classes of animals that zoos will exhibit for which dietary D may be a problematic solution to maintaining bone health.

The ideal solution is to give animals access to unfiltered sunlight. This ideal solution is neither always practical nor possible, however. Many animals must be housed in enclosed exhibits. Thus any sunlight they will be exposed to passes through potentially UV-B blocking materials. Most forms of glass and plastic are not transparent to UV-B radiation, however commercially available UV-B transparent materials are marketed. Zoos should give thought to testing these materials for use in enclosed exhibits.

The use of artificial UV-B light sources could be effective under certain circumstances. However, the potential eye and skin hazards of high intensity UV light for both animals and keepers must be considered, as well as the rapid decline in UV intensity that occurs with distance from the source. Episodic exposure to high intensity UV-B should be tested, as this strategy might be effective for certain species. Clinical intervention with the administration of either oral or injected vitamin D is often disruptive and labor intensive, but may be the only practical approach for nursing infants of some primate species it is not possible to ensure adequate exposure of the infant to UV-B.

\section{Symptoms}

Osteoporosis: Osteoporosis is a disease well known in humans as well as in certain captive animals. This is a thinning of the bone matrix as more calcium is resorbed into the blood than is deposited into the bone. Rather than being caused strictly by a deficiency in calcium or excess of phosphorus, true osteoporosis may be related to protein deficiency (both dietary and due to prolonged anorexia) or through disuse of bones. This may be as a result of being confined in too-small spaces, or enforced inactivity such as that experienced when fractures have been immobilized, or as a result of long-term paralysis. Bones become brittle, light, and easily broken.

Osteomalacia: This is softening of the bone and decreased bone density due to decreased mineralization. The body tries to compensate by depositing bone material at the sites of greatest tension. Folding fractures and bowed bones are common.

Rickets: Rickets is similar to osteomalacia, and occurs in the young. The differences are most noticeable in $\mathrm{x}$-rays as it affects the bones in different ways. Bowing of the long bones, sometimes severely deformed long bones, is the most common outward signs of rickets.

\section{Secondary Nutritional hyperparathyroidism}

This is an important metabolic disturbance in captive reptiles mainly due to nutritional disorder caused either by deficiency of dietary calcium and vitamin $\mathrm{D}_{3}$ or UV-b radiation [6]. The low levels of serum calcium stimulate the production of PTH (parathyroid hormone), which causes the bones to release calcium and phosphate, as well as stimulating 1,25-dihydroxycolecalciferol, which increases calcium absorption in the gut. The result is bones stripped of calcium, with insufficient calcium available to replace the calcium lost from the bone, and so the bone weakens. This results in osteomalacia in adults and rickets in the young. Calcium deficiencies may be caused by kidney, intestinal and liver diseases as well as by dietary deficiencies. Diseases which affect the way in which nutrients are extracted from food and metabolized may ultimately affect how calcium is processed in the body.

\section{Metabolic bone disease in reptiles}

Metabolic bone disease is a serious and widespread cause of suffering and death among captive reptiles. This is in majority cases due to improper ratio of calcium-phosphorus in the captive diet and lack of exposure of sufficient sunlight. The three most important nutrients for bone development are calcium, phosphorus and vitaminD ${ }_{3}$, which form a triad of nutrients that work with each other. All three nutrients are necessary and must be in roughly the correct proportion to each other.

\section{The calcium - phosphorus ratio}

The ratio of calcium to phosphorus is more important than absolute amounts. For example, doubling the calcium in an animal's 
diet could cause it to develop metabolic bone disease unless phosphorus is raised as well. On the other hand, cutting the amount of calcium in half might have no effect if phosphorus is lowered accordingly. While there are differences between individuals, all vertebrates, particularly growing animals, are at risk of metabolic bone disease if the ratio of calcium to phosphorus in their overall diet falls below 1:1 or exceeds 2:1. There are several food items commonly fed to captive reptiles that can cause the calciumphosphorus ratio to fall outside the safe 1:1 to 2:1 range, which are following:

i. It is always tempting to feed an animal more and more of a food items it likes, and most herbivorous and omnivorous reptiles like fruit. Unfortunately, fruit is low in calcium and if it becomes a large portion of the animal's diet, it may drive the calcium phosphorus ratio to less than 1:1.

ii. Many carnivorous reptiles like insects, particularly crickets, meal worms and wax worms, which usually are low in calcium.

\section{Calcium and vitamin $\mathrm{D}_{3}$}

Vitamin $\mathrm{D}_{3}$ is necessary components to perform a vital function with calcium in laying down the bone structure in a vertebrate's body. Like calcium and phosphorus, an animal can have too much $\mathrm{D}_{3}$ or too little $\mathrm{D}_{3}$. When there is too little $\mathrm{D}_{3}$ (the common problem), calcium is not deposited properly for bone formation. When there is too much (less common), calcium deposits can form in the body where they should not (soft-tissue calcification) and damage the health of the animal. Animal food items such as crickets, pinkies, etc. are rich in vitamin $D_{3}$ so carnivorous reptiles usually get plenty of $D_{3}$ in their diet. On the other hand, plant food items do not contain $\mathrm{D}_{3}$ and have varying amounts of $D 2$, so herbivorous and omnivorous reptiles have to synthesize $\mathrm{D}_{3}$ in their own bodies. By basking in the sun, the animal uses ultraviolet light to convert sterol precursors to vitamin $\mathrm{D}_{3}$. This conversion process works well in wild reptiles, while very little or does not happen in captive reptiles.

i. Generally, it is believed that captive reptiles need a lot of sunlight to synthesize enough $\mathrm{D}_{3}$ for avoiding metabolic bone disease. But due to captivity of reptiles, could not get enough sunlight to prevent metabolic bone disease.

ii. The $\mathrm{D}_{3}$ synthesis process requires UV-B radiation in the 280-315 $\mathrm{nm}$ range and this kind of sunlight does not travel well through glass or other similar transparent barriers. Hence, captive reptile must be left outdoors to synthesize enough $\mathrm{D}_{3}$.

iii. Ultraviolet lamps can help, but the amount of radiation in the $280-315 \mathrm{~nm}$ range that be safely provided by these lamps is often so small that they cannot reliably serve as a substitute for natural sunlight.

\section{Common ailments of tortoises in captivity}

The soft or deformed shell is the common ailment of pet tortoises. This is due to a lack of calcium in the diet or a problem absorbing dietary calcium. Tortoises need exposure to ultraviolet radiation $A$ and $B$ (UVA/B) in order to manufacture vitamin $D_{3}$ from vitamin D2. Vitamin $\mathrm{D}_{3}$ is necessary for calcium in the diet to be absorbed through the gastrointestinal tract. In the wild, the sun provides ultraviolet light. Wild tortoises bask 8 to 14 hours/day and receive the necessary UVA/B rays in order to absorb calcium from food. Sunlight that shines through a glass or plastic window or cage is blocked from providing the necessary radiation. Tortoises fed a diet low in calcium, or those who cannot absorb calcium well, usually first show a soft shell, especially if they are a juvenile. Baby tortoises naturally have a semi-soft shell after hatching, but it usually hardeners by 6 to 8 months of age. In calcium-deficient tortoises, the tortoise grows, but the shell does not. This causes the shell to become deformed and often the tortoise looks too big for its shell. It become weak, and eventually has trouble walking, due to soft bones. The soft bones can easily fracture, causing further problems. If untreated, the tortoise will eventual die. This is often a slow, painful death.

\section{Respiratory Infection}

Respiratory infections are common in pet tortoises, especially in wild-caught individuals sold in the pet trade. Being shipped from their native lands and held in less-than-desirable circumstances causes a great deal of stress on the tortoise. Add to this that often the poor village people collecting them may lack the means (heated houses, running water, available food, sanitation, etc) to house them properly before shipment ever takes place. Many times tortoises are held in great numbers in small un-heated enclosures for weeks to months, with little food or water. Tortoises shows lethargy, ocular and nasal discharge, weight loss (a healthy tortoise should feel heavy for its size, like its shell is filled with water), with increased respiratory rates and wheezing. These ill tortoises will often refuse food despite being given proper care and an environment.

\section{Symptoms}

Depression: Affected reptiles will become quiet, depressed and listless, often refusing to move at all or sleeping excessively. The skin of brightly colored animals such as iguanas chameleons may also change and become dark and drab.

Swelling of the legs: As the bones and joints of the legs become thinner and weaker, the reptile will experience pain and difficulty in walking, and their movement may become more awkward, stiff or uncoordinated. Lizards such as bearded dragons and geckos may be unable to hold their weight and instead will drag themselves along the floor. Arboreal lizards may begin to spend more time sitting on the floor of their vivarium as climbing and holding on to branches becomes impossible.

Swelling of the jaw: As the jaw bones become soft, they often become inflamed and painful, causing the reptile to stop eating and lose weight rapidly.

Fits, muscle tremors and seizures: The lack of calcium in the blood will eventually cause a break down in the correct functioning 
of the nervous and muscular systems, resulting in uncontrollable shivering and twitching of the muscles, as well as a complete loss of balance and coordination.

Distortion, dislocations and fractures of the long bones: Softening of the skeleton as calcium is stripped away causes the long bones of the radius, ulna and femur to become brittle, often causing them to suffer from folding fractures or permanent bending and twisting. The joints of the hips, shoulders and elbows may also become dislocated.

Deformity of the skull and jaws: The upper jaw and dome of the skull may become contracted, causing the tongue and lower jaw to protrude become spongy lower jaw bone and putting pressure on the brain.

Twisting and distortion of the spinal vertebrae: It causes the backbone to appear kinked, hunched or lumpy, and sometimes causing paralysis as the spinal cord is crushed or pinched.

Stomach bloat and gut rot: As the muscles that control the digestive tract shut down, any food remaining in the stomach or intestines will begin to ferment and rot, resulting in an accumulation of gas and toxin. In tortoises, the carapace and plastron of the shell will become soft, twisted and flattened, putting pressure on the internal organs.

Eventually, after a period of several weeks, the reptile suffering from advanced stage MBD will succumb to its symptoms and die, often as a result of a heart attack as the cardiac muscle is unable to beat properly without adequate calcium or from asphyxiation as the rib cage collapses, so making breathing impossible.

\section{Metabolic Bone Disease in Captive Birds}

The captive birds unfortunately received a poor diet with inadequate UV light, because of seed-based mixes diets traditionally used for feeding which are low levels of both vitamin $\mathrm{D}_{3}$ and calcium. In addition, they can contain high levels of phosphorus that can bind the calcium in phytate complexes.10 Chronic deficiency of vitamin D would be expected to lead to hypocalcemia and secondary hyperparathyroidism. Vitamin D is obtained by birds directly from the diet and by the action of UV-B on vitamin D precursors in the cutaneous tissues. Either a deficiency in dietary vitamin D or lack of available UV-B light would be expected to produce a vitamin D-deficient bird, potentially leading to subsequent problems with calcium metabolism. Which leads to hypocalcemia, is a common syndrome in avian species $[7,8]$

There are several factors which are responsible for the development of MBD in birds which includes:

a. Poor (nutrient imbalanced) diets fed to birds. Poor diets (the classic all seed diet) is commonly blamed for MBD in birds.

b. Lack of exposure to natural unfiltered sunshine because of birds kept entirely indoors. This causes insufficient synthesis of vitamin D in bird's skin. c. Exposure to toxins or certain foods that competitively block the absorption of select nutrients (like calcium), cause increased elimination of calcium from the kidneys or that binds with calcium and other bone building nutrients in the diet (and subsequently prevent their absorption). The most common is excessive feeding of spinach and plant produces fruits which contain high oxalate result in either decreased absorption and/ or urinary excretion of calcium (that is why they are sometimes associated with calcium oxalate kidney or urinary bladder stones).

d. Prolonged illness that results in decreased intestinal absorption of bone related nutrients. This can be something as simple as chronic diarrhea that decreases the amount of time foods have in the gut to be absorbed. More often, it is illnesses that affect the proventriculus/ventriculus (stomach) like proventriculuar dilatation syndrome or avian gastric yeast or various forms of liver and pancreatic disease that may prevent absorption of multiple nutrients. Primarily parathyroid diseases (which affect calcium and phosphorous balance) are considered rare in pet birds. However, we do commonly see secondary parathyroid disease as a result of MBD in pet birds $[9,10]$.

\section{Conclusion}

Metabolic bone diseases of captive animals are most important clinical manifestation occurred as a result of nutritional disorder either caused by deficiency of dietary calcium and vitamin $\mathrm{D}_{3}$ or inadequate exposure to UV-b radiation.

\section{References}

1. Marx SJ, Jones G, Weinstein RS, Chrouse GP, Renquist DM (1989) Differences in mineral metabolism among nonhuman primates receiving diets with only vitamin D3 or only vitamin D2. J Clin Endocrinol Metab 69(6): 1282-1290.

2. Hollis BW, Roos BA, Draper HH, Lambert PW (1981) Vitamin D and its I in human and bovine milk. Journal of Nutrition 111: 1240-1248.

3. Anderson JJB, Toverud SU (1994) Diet and Vitamin D: A review with an emphasis on human function. J Nutri Biochem 5(2): 58-65.

4. Fraser DR (1995) Vitamin D. The lancet 345(8942): 104-107.

5. Kenny D, Cambre RC, Lewandowski A, Pelto JA, Irlbeck NA, et al. (1993) Suspected vitamin D3 toxicity inh - (Cuniculus paca) and agoutis (Dasyprocta aguti). Journal of zoo and wildlife medicine 24(2): 129-139.

6. Mans C, Braun J (2014) Updates on common nutritional disorders of captive reptiles. Vet Clin North Am Exot Anim Pract 17(3): 369-395.

7. Dacke GC (2000) Sturkie's Avian Physiology ( $5^{\text {th }}$ edn), In: Whittow GC (Ed.), London, UK, Academic Press 472-485.

8. Stanford MD (2003) Measurement of 25 hydroxycholecalciferol in grey parrots: The effect of diet. Proc Euro Assoc Avian Vet.

9. Blood DC, Stoddert VP (1988) Baillière's Comprehensive Veterinary Dictionary. In: Baillière \& Tindall (Eds.), London, UK 1124 p.

10. Fowler ME (1986) Zoo \& Wild Animal Medicine. WB Saunders Company, Philadelphia PA, United States p. 1087. 
Creative Commons Attribution 4.0 International License

For possible submissions Click Here

Submit Article

\section{APDV $\quad$ Approaches in Poultry, Dairy \& Veterinary Sciences \\ Benefits of Publishing with us}

- High-level peer review and editorial services

- Freely accessible online immediately upon publication

- Authors retain the copyright to their work

- Licensing it under a Creative Commons license

- Visibility through different online platforms 